\title{
Politique
}

Politique

\section{Management public : comprendre et gérer les institutions de l'État sous la direction de Roland Parenteau, Sainte-Foy, Les Presses de l’Université du Québec, 1992, 640 p.}

\section{Gilles Bouchard}

Numéro 22, automne 1992

URI : https://id.erudit.org/iderudit/040739ar

DOI : https://doi.org/10.7202/040739ar

Aller au sommaire du numéro

Éditeur(s)

Société québécoise de science politique

ISSN

0711-608X (imprimé)

1918-6584 (numérique)

Découvrir la revue

Citer ce compte rendu

Bouchard, G. (1992). Compte rendu de [Management public : comprendre et gérer les institutions de l'État sous la direction de Roland Parenteau, Sainte-Foy, Les Presses de l'Université du Québec, 1992, 640 p.] Politique, (22), 161-163. https://doi.org/10.7202/040739ar d'utilisation que vous pouvez consulter en ligne.

https://apropos.erudit.org/fr/usagers/politique-dutilisation/ 
Management public : comprendre et gérer les institutions de I'État

sous la direction de Roland Parenteau, Sainte-Foy, Les Presses de I'Université du Québec, 1992, 640 p.

Un titre accrocheur, une table des matières qui ne semble pas correspondre au titre et une présentation du directeur expliquant le choix du titre, voilà les premières pages de cet ouvrage de plus de six cents pages regroupant vingt-sept articles, signés pour la plupart par des professeurs de l'École nationale d'administration publique. La justification de M. Roland Parenteau, directeur de la publication, concernant le choix du titre $\mathrm{m}$ 'a ramené près de vingt ans en arrière. À cette époque, nos professeurs d'administration publique annonçaient fièrement qu'après s'être préoccupés un peu trop longuement dans les années cinquante du "comment faire", nous allions enfin nous intéresser à une question qui apparaissait à tous plus fondamentale, soit le "quoi faire». Aujourd'hui, M. Parenteau tient le discours inverse. Selon lui, le management public en mettant l'accent sur le "comment faire" représente un progrès indéniable sur l'administration publique, une discipline dominée par des politicologues s'intéressant essentiellement au "quoi faire". Et on dira que rien ne change!

L'ouvrage est divisé en cinq parties. Une première traite de la nature du management public. Les quatre premiers chapitres en 
particulier essaient de positionner le management public entre d'une part le management privé et d'autre part l'administration publique. Si l'on réussit sans trop de mal à démarquer le management public du management privé, les deux auteurs qui essaient de distinguer administration publique et management public ne sont, à la fois, guère convaincants ni convaincus. L'article de Gérard Bergeron est particulièrement intéressant à cet égard. Sans dénoncer explicitement le terme management public, il se demande si l'emploi de ce terme n'est pas une simple mode ou l'illustration de ce qu'il appelle un glissement inconsidéré du domaine privé au public.

La seconde partie aborde la question des managers publics. On les décrit, on dit ce qu'ils font, comment ils peuvent devenir compétents et comment ils doivent se comporter (éthique). La troisième partie examine le lien entre politique et management. Pour un ouvrage qui veut se démarquer de la science politique, cette partie peut paraître incongrue, car son thème réel est politique et administration. On y traite de sujets aussi traditionnels que l'imputabilité et les relations sous-ministres et appareil politique. La quatrième partie fait place aux processus et aux systèmes de gestion. Planification stratégique côtoie analyse des politiques alors que marketing gouvernemental précède gestion des personnes. Enfin, une dernière partie, trop courte au goût de $M$. Roland Parenteau, aborde la question de la performance du secteur public en parlant de qualité, de productivité et de décroissance.

Que ce soit un ouvrage de management public ou d'administration publique, il s'agit, disons-le tout de suite, d'un excellent ouvrage de référence. Comme son directeur le précise en introduction, il ne s'agit pas d'un manuel de base pour un cours spécifique, car l'ouvrage ne couvre pas systématiquement tous les aspects de la discipline et on n'y retrouve guère de fil conducteur ou même d'unité de pensée. Par contre, comme ouvrage de référence, $c^{\prime}$ est un volume qui contient plusieurs articles qui font le point sur divers thèmes importants en se basant sur la littérature la plus récente. On y retrouve aussi une pluralité de perspectives allant du upublic choice» au post-modernisme, en passant par les divers courants du management. L'administration publique québécoise est bien sûr la référence obligée de presque tous les auteurs, ce qui peut rendre le volume moins intéressant pour un lecteur étranger. Les bibliographies de chacun des chapitres donnent au lecteur une liste imposante d'ouvrages récents dont une bonne part est de langue française. Parmi les meilleurs articles, citons ceux d'Adrien Payette sur les éléments pour une 
conception du management public et sur l'éthique, celui de Bourgault-Dion sur les sous-ministres, ceux de Maurice Patry sur I'imputabilité, celui de Gérard Éthier sur la qualité des services et de Michel Paquin sur la décroissance. C'est un livre qui figurera avec profit dans la bibliothèque de ceux et celles qui s'intéressent au management public, pour ne pas dire à l'administration publique.

Gilles Bouchard 